\title{
Generalization of the Cyclic Pursuit Problem
}

\author{
A. Sinha and D. Ghose
}

\begin{abstract}
In this paper, some generalizations of the problem of formation of a group of autonomous mobile agents under cyclic pursuit is studied. Cyclic pursuit is a simple distributed control law, in which the agent $i$ pursues agent $i+1$ modulo $n$. Each agent is subjected to a nonholonomic constraint. The necessary conditions for equilibrium formation to occur among a group of agents with different speeds and controller gains is discussed. These results generalize equal speed and equal controller gain results available in the literature.
\end{abstract}

\section{INTRODUCTION}

This paper deals with the problem of cyclic pursuit in a multi-vehicle system. Multi-vehicle systems are groups of autonomous mobile agents used in search and surveillance tasks, rescue missions, space and oceanic explorations, and other automated collaborative operations. Cyclic pursuit uses simple local interaction between these vehicles to obtain desired global behaviour.

The pursuit strategies are designed to mimic the behaviour of biological organisms like dogs, birds, ants, or beetles. They are commonly referred to as the 'bugs' problem. Bruckstein et al. [1] modelled the behavior of ants, crickets and frogs with continuous and discrete pursuit laws and examined the possible evolution of global behaviour such as the convergence to a point, collision, limit points, or periodic motion. Convergence to a point in linear pursuit is the starting point to the analysis of achievable global formation among a group of autonomous mobile agents as discussed in [2]. The paper [2] also deals with the evolution of the formation of these agents with respect to the possibility of collision. Kinematics of agents with single holonomic constraint is discussed in [3] and [4]. The equilibrium and stability of identical agents with this motion constraint is dealt in these papers as well as in [5]. The mathematics governing cyclic pursuit are studied in [6] - [10]. Generalization of the linear version of the cyclic pursuit has been presented in [11].

This work has been inspired by the problem addressed in Marshall et al. [5]. They considered $n$ identical autonomous mobile agents in cyclic pursuit with a single nonholonomic constraint. The agents are assumed to have same speed and controller gains. In our paper, a more general case is discussed where the speeds and controller gains for different

The authors would like to acknowledge the financial support received from the IISc-DRDO Program on Advanced Research in Mathematical Engineering.

A. Sinha is a Graduate Student in the Department of Aerospace Engineering at the Indian Institute of Science, Bangalore, India asinha@aero.iisc.ernet.in

D. Ghose is a Professor in the Department of Aerospace Engineering at the Indian Institute of Science, Bangalore, India dghosedaero.iisc.ernet.in

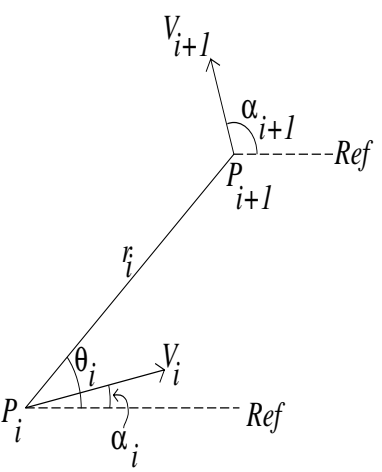

Fig. 1. Basic formation geometry

agents may vary, thus giving rise to a heterogenous system of agents. In such a case, determination of conditions under which the system converges to an equilibrium becomes more complicated. The conditions necessary for such a convergence is analyzed here.

\section{EQUATIONS OF CYCLIC PURSUIT}

Nonlinear cyclic pursuit is an extension of the classical $n$ bugs problem or linear pursuit. Each bug is modelled as a point mass autonomous mobile agent. The agents are ordered from 1 to $n$, and the agent $i$ follows agent $i+1$, modulo $n$.

Each agent has a constant velocity $V_{i}$, and orientation $\alpha_{i}$ (variable), with respect to a fixed reference (Fig. 1). The distance between the $i^{t h}$ and $i+1^{t h}$ agent is $r_{i}$ and the angle from the reference to the line of sight (LOS) from agents $i$ to $i+1$ is given by $\theta_{i}$. The control input to the $i^{t h}$ agent is the lateral acceleration $a_{i}$ which is given as:

$$
a_{i}=k_{i} \phi_{i}
$$

where (with reference to Fig. 2)

$$
\begin{array}{lr}
\alpha_{i}+\phi_{i}-\theta_{i}=0 & \text { if } 0 \leq \alpha_{\mathrm{i}} \leq \theta_{\mathrm{i}} \\
\alpha_{i}+\phi_{i}-\theta_{i}=2 \pi & \text { otherwise }
\end{array}
$$

where $k_{i}$ is the controller gain. The lateral acceleration, so defined, guarantees that all the agents move in the counter clockwise direction.

Thus, the kinematics of the $i^{t h}$ agent is given as follows:

$$
\begin{aligned}
\dot{r_{i}} & =V_{i+1} \cos \left(\alpha_{i+1}-\theta_{i}\right)-V_{i} \cos \left(\alpha_{i}-\theta_{i}\right) \\
r_{i} \dot{\theta}_{i} & =V_{i+1} \sin \left(\alpha_{i+1}-\theta_{i}\right)-V_{i} \sin \left(\alpha_{i}-\theta_{i}\right) \\
\dot{\alpha_{i}} & =\frac{a_{i}}{V_{i}}=k_{i} \frac{\phi_{i}}{V_{i}}
\end{aligned}
$$



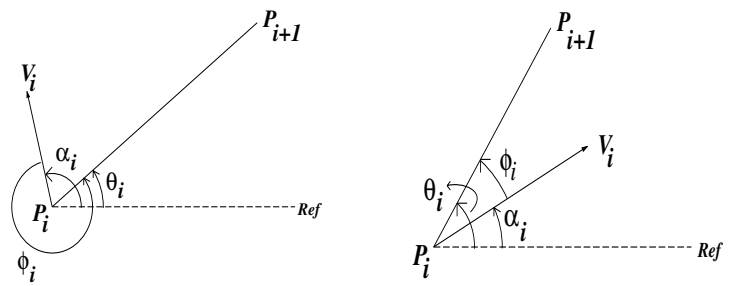

Fig. 2. Representation of the angles with respect to a fixed reference

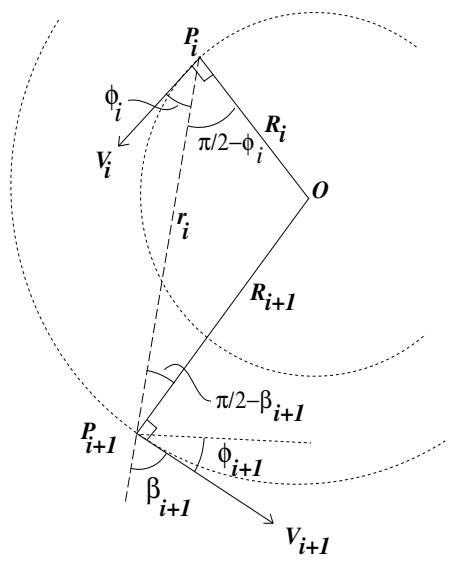

Fig. 3. Multi-vehicle formation with circular trajectory

\section{ANALYSIS FOR POSSIBLE FORMATIONS}

For a system of $n$ agents, with kinematics (2) - (4), equilibrium is said to have been achieved if the relative position of the agents do not change with time. Therefore, at equilibrium,

$$
\begin{aligned}
\dot{r}_{i} & =0 \\
\dot{\phi}_{i} & =0
\end{aligned}
$$

which is similar to the condition of equilibrium considered in [5]. Now, let us assume that the $n$ agents eventually converge to a equilibrium formation. Then we can state the following theorem.

Theorem 1. At equilibrium, a system of $n$ agents, with kinematics (2)-(4), move in concentric circles with equal angular velocities.

Proof. $\dot{\phi}_{i}=0 \Rightarrow \phi_{i}=$ constant $\Rightarrow a_{i}=$ constant. Therefore, any agent $i$, having constant linear speed $V_{i}$ and constant lateral acceleration $a_{i}$, will move in a circular trajectory. Considering Fig. 3, let the position of the $i^{\text {th }}$ agent be $P_{i}$ and the center of the circle traversed by it be O. Let, $R_{i}=d$ where $d$ is some constant. Again, $\phi_{i}=$ constant implies $\angle O P_{i} P_{i+1}=\left(90-\phi_{i}\right)$ is a constant. Therefore, the position of $i+1^{\text {th }}$ agent should be on the line $P_{i} P_{i+1}$ or its extension. The point at which $i+1^{t h}$ agent lies on $P_{i} P_{i+1}$ is determined from $\dot{r}_{i}=0$ which implies that $r_{i}=$ constant. Therefore, $\triangle O P_{i} P_{i+1}$ forms a rigid triangle. Thus, agent $i+1$ also has the center of its circular trajectory at $O$. Again, since the configuration of the $\triangle O P_{i} P_{i+1}$ remains rigid at equilibrium, we have $\omega_{i}=\omega_{i+1}$, where $\omega_{i}$ is the rate of rotation of $O P_{i}$. Hence, all agents move in concentric circles with equal angular velocity.

Let, the radius of the circle traversed by the first agent at equilibrium be $R_{1}=\rho$. Since the angular velocity is the same for all the agents, we have

$$
\omega_{i}=\omega_{i+1} \quad \Rightarrow \frac{V_{i}}{R_{i}}=\frac{V_{i+1}}{R_{i+1}} \quad \Rightarrow R_{i}=\frac{V_{i} \rho}{V_{1}}
$$

Now,

$$
a_{i}=\frac{V_{i}^{2}}{R_{i}}=k_{i} \phi_{i} \quad \Rightarrow \phi_{i}=\frac{V_{i} V_{1}}{k_{i} \rho}
$$

Again, from $\triangle O P_{i} P_{i+1}$,

$$
\begin{array}{r}
\frac{R_{i+1}}{R_{i}}=\frac{\sin \left(90-\phi_{i}\right)}{\sin \left(90-\beta_{i+1}\right)} \\
\Rightarrow \beta_{i+1}=\cos ^{-1}\left[\frac{V_{i}}{V_{i+1}} \cos \left\{\frac{V_{1} V_{i}}{k_{i} \rho}\right\}\right]
\end{array}
$$

Let the velocity ratio $\frac{V_{i+1}}{V_{i}}=\gamma_{i+1}$. Therefore,

$$
\sum_{i=1}^{n}\left(\phi_{i}+\beta_{i}\right)=\sum_{i=1}^{n}\left[\frac{V_{i} V_{1}}{k_{i} \rho}+\cos ^{-1}\left\{\frac{1}{\gamma_{i}} \cos \left(\frac{V_{i-1} V_{1}}{k_{i-1} \rho}\right)\right\}\right]
$$

where the subscript indices are modulo $n$.

Now, consider any $n$ sided polygon (not necessary regular), a part of which is shown in Fig. 4. Each node $i$ represents the position of the agent $i$ and the vector from that node represents the velocity of the $i^{t h}$ agent. Thus the angle $\left(\phi_{i}+\beta_{i}\right)$ is measured counter-clockwise from the extension of the line $P_{i-1} P_{i}$ to the line $P_{i} P_{i+1}$, as shown in Fig. 4. Therefore, considering all possible polygonal topologies for a given $n, \sum\left(\phi_{i}+\beta_{i}\right)=2 q \pi$ where $q=$ $1,2, \cdots,(n-1)$. The $q$ considered here is similar to the variable $d$ used in Definition 2 in [5]. Thus,

$$
\sum_{i=1}^{n}\left[\frac{V_{i} V_{1}}{k_{i} \rho}+\cos ^{-1}\left\{\frac{1}{\gamma_{i}} \cos \left(\frac{V_{i-1} V_{1}}{k_{i-1} \rho}\right)\right\}\right]=2 q \pi
$$

where the subscript indices are modulo $n$.

Hence, if a system of $n$ vehicles with arbitrary speeds and controller gains $k_{i}$ attains equilibrium, Eqn (8) must be satisfied for some $\rho$ and for some $q=1,2, \cdots, n-1$. We will attempt to refine this condition further by analysing Eqn (8).

For a given $q$, Eqn. (8) is a function of $\rho$ only. Therefore, the values of $\rho$ that satisfies Eqn. (8) gives the radius of the circle of the first agent. The radius for the other agents can be obtained from Eqn. (5). 


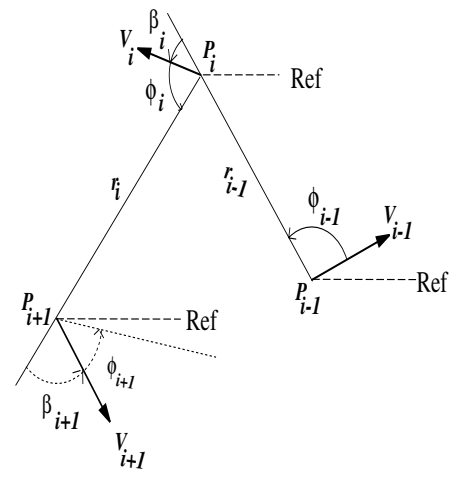

Fig. 4. Angle calculation for a general polygon of $n$ sides

Let us denote the LHS of Eqn. (8) by

$$
f(\rho)=\sum_{i=1}^{n}\left[\frac{V_{i} V_{1}}{k_{i} \rho}+\cos ^{-1}\left\{\frac{1}{\gamma_{i}} \cos \left(\frac{V_{i-1} V_{1}}{k_{i-1} \rho}\right)\right\}\right]
$$

Theorem 2. Necessary condition for equilibrium. Consider an $n$ agent system with kinematics given by (2)-(4). Equilibrium formation can be obtained if there exist a $\rho$ such that,

$$
\max _{i \in\left\{j: V_{j}>V_{j+1}\right\}} a_{i} \leq \frac{1}{\rho} \leq \min _{i \epsilon\left\{j: V_{j}>V_{j+1}\right\}} b_{i}
$$

where,

$$
\begin{array}{r}
a_{i}=\left[m \pi+\cos ^{-1}\left(\gamma_{i+1}\right)\right] \frac{k_{i}}{V_{i} V_{1}} \\
b_{i}=\left[(m+1) \pi-\cos ^{-1}\left(\gamma_{i+1}\right)\right] \frac{k_{i}}{V_{i} V_{1}}
\end{array}
$$

and $m=0,1$.

Proof. Eqn. (8) exists if and only if the argument of the $\cos ^{-1}$ term is in $[-1,1]$, i.e.,

$$
\left|\cos \left(\frac{V_{i} V_{1}}{k_{i} \rho}\right)\right| \leq \gamma_{i+1}, \quad \forall i
$$

Let $X_{1}=\left\{i: V_{i}>V_{i+1}\right\}$ and $X_{2}=\left\{i: V_{i} \leq V_{i+1}\right\}$. Note that both $X_{1}$ and $X_{2}$ are nonempty sets. For all $i \epsilon X_{2}$, (10) is always satisfied irrespective of the values of $\rho$. For a given $i \epsilon X_{1}$, the range of values $\rho_{i}$ that $\rho$ can take is

$$
\hat{a}_{i} \leq \frac{V_{1} V_{i}}{k_{i} \rho_{i}} \leq \hat{b}_{i}
$$

where,

$$
\begin{array}{r}
\hat{a}_{i}=\left[m \pi+\cos ^{-1}\left(\gamma_{i+1}\right)\right] \\
\hat{b}_{i}=\left[(m+1) \pi-\cos ^{-1}\left(\gamma_{i+1}\right)\right]
\end{array}
$$

where $m=0, \pm 1, \pm 2, \cdots$. Now, $\frac{V_{i} V_{1}}{k_{i} \rho_{i}}=\phi_{i}$. The range of $\phi_{i}$ is shown in Fig. 6. From figure 5, it is evident that the possible values of $m$ are 0 or 1 .

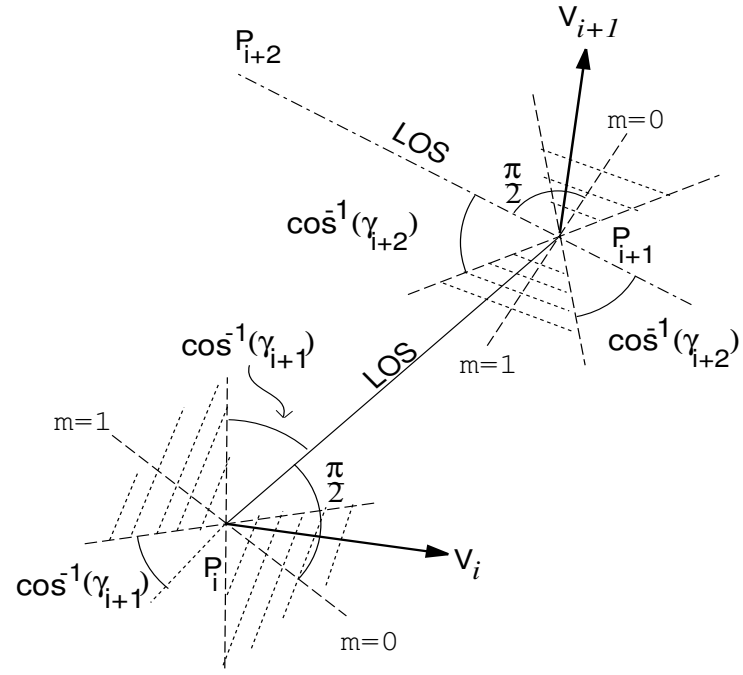

Fig. 5. Representation of the range of $\phi$ in polar coordinate
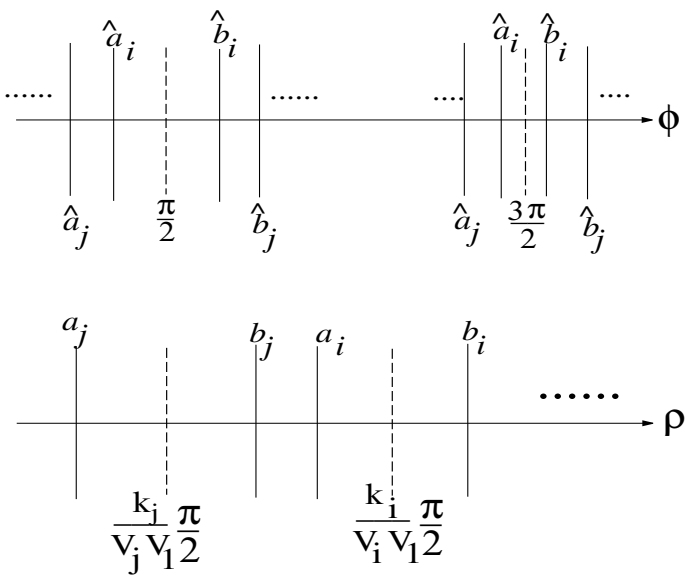

Fig. 6. General representation of the ranges of $\phi$ and $\rho$ for all agents

Now, let $\mathcal{R}_{i}=\left\{\rho_{i}: \rho_{i}\right.$ satisfies (11) $\}$. $\mathcal{R}_{i}$ has a center at $\frac{k_{i}}{V_{i} V_{1}}\left(\frac{2 m+1}{2} \pi\right)$ and a spread of $\left(\pi-2 \frac{k_{i}}{V_{i} V_{1}} \cos ^{-1}\left(\gamma_{i+1}\right)\right)$. Since both the center and spread of $\mathcal{R}_{i}$ are functions of $V_{i}, V_{i+1}$ and $k_{i}$, therefore they are not constant for different $i$ 's. Similarly, the distance between the centers are also not constant.

There will exist some value of $\rho$ that will satisfy Eqn. (11) for all $i \epsilon X$ if

$$
\bigcap_{i \in X_{1}} \mathcal{R}_{i} \neq \emptyset
$$

This implies that some $\rho$ should satisfy (9) for all $i \epsilon X_{1}$.

Special case. We may consider a special case in which $V_{i}=$ $V$ and $k_{i}=k$, for all $i$, which is the assumption made in [5]. Then Eqn. (8) reduces to

$$
\frac{2 V^{2} n}{k \rho}=2 q \pi \quad \Rightarrow \rho=\frac{V^{2} n}{q k \pi}
$$

Thus, all the agents will move in a fixed circle of radius $\frac{V^{2} n}{q \pi k}$ at equilibrium. This is the same as the result obtained 


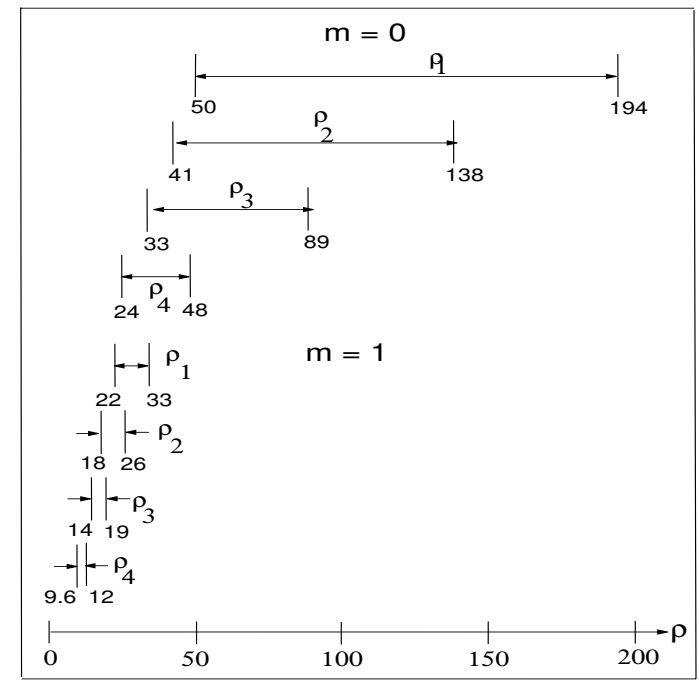

Fig. 7. Range of $\rho_{i}$ for Case 1

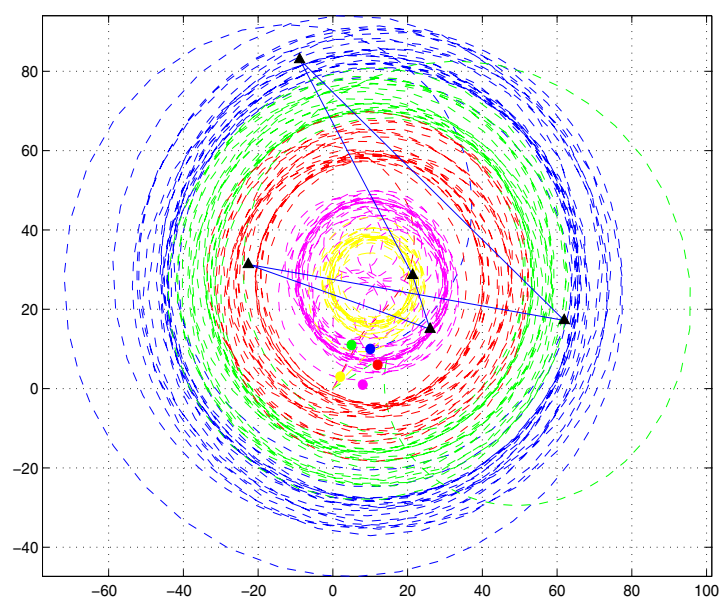

Fig. 8. Trajectories of $n=5$ agents for case 1 (circle: initial position, triangle: final position of the UAVs)

in [5], the apparent difference in the exact expression is due to the choice of the controller gain which in [5] is defined as $k V$. Again, from (6), $\phi=\frac{q \pi}{n}$, which is the same as in [5].

\section{Simulation Results}

Case 1: Consider a system of $n=5$ agents. The velocities of the agents are $V=\left[\begin{array}{lllll}25 & 20 & 15 & 10 & 5\end{array}\right]$ while the gain is taken to be the same for all, i.e., $k_{i}=5$ for all $i$. So, $X_{1}=\left\{\begin{array}{llll}1 & 2 & 3 & 4\end{array}\right\}$. Note that all values of $\rho_{5}$ satisfies (10). The range of values $\rho_{i}$ that satisfies Eqn. (11) are shown in Fig. 7. For both $m=0$ and $1, \bigcap_{i \in X_{1}} \rho_{i}=\emptyset$. The simulation result (Fig. 8) also shows that the system does not have an equilibrium.

Case 2: Consider the previous example with all the values same except for $V_{5}=6$. Again, $\rho_{5}$ automatically satisfies (10) for all values. But now, for $m=0$, the range for $\rho_{1}$,

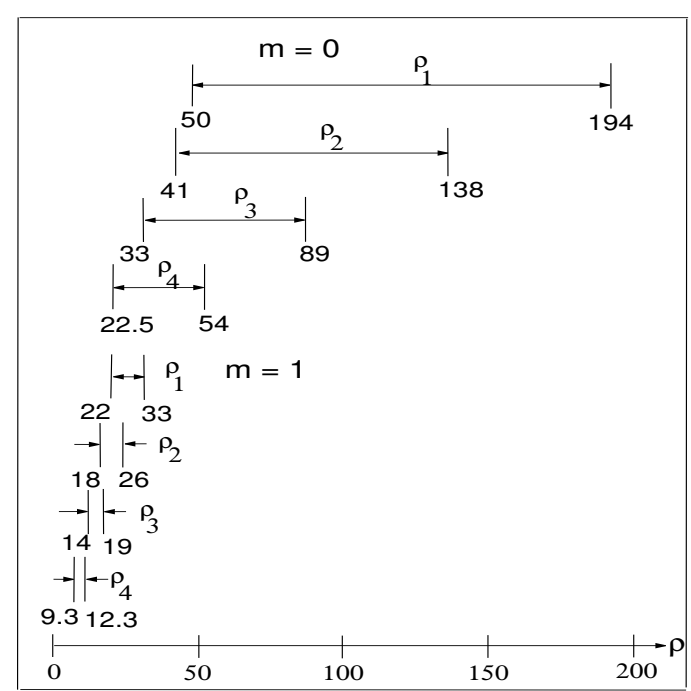

Fig. 9. Range of $\rho_{i}$ for Case 2

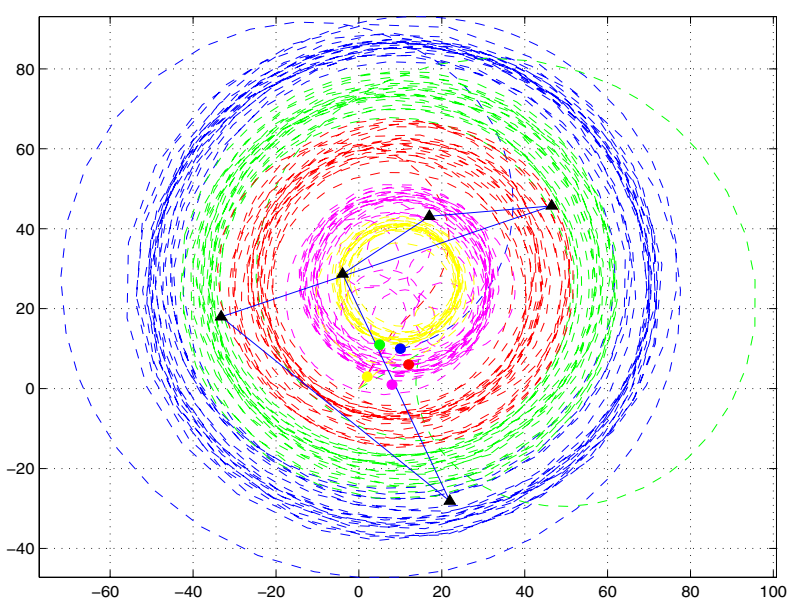

Fig. 10. Trajectories of $n=5$ agents for Case 2 (circle: initial position, triangle: final position of the UAVs)

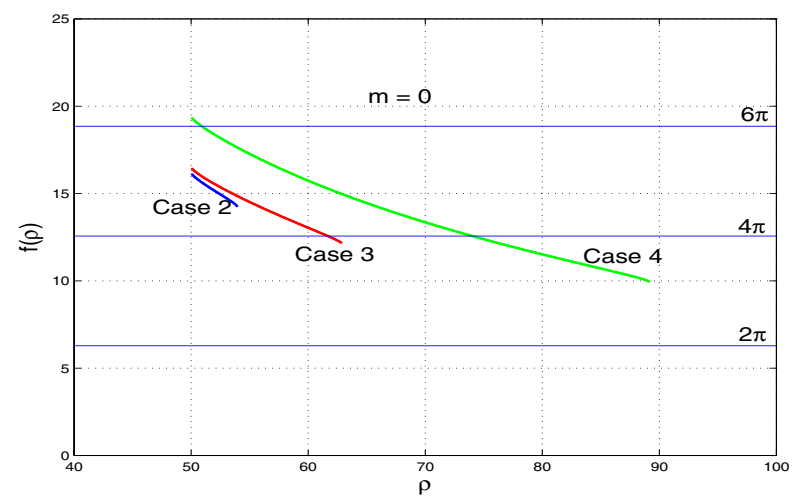

Fig. 11. The roots of Eqn. (8) for Cases 2, 3, and 4 


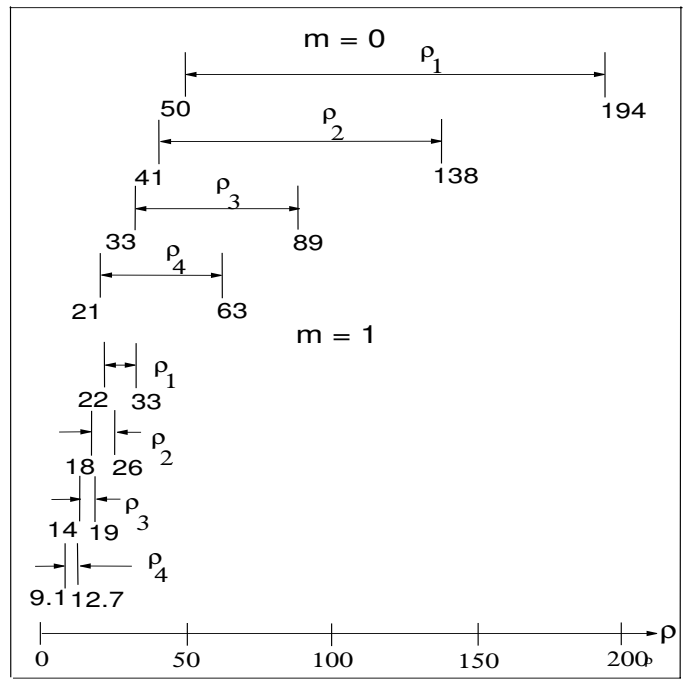

Fig. 12. Range of $\rho_{i}$ for Case 3

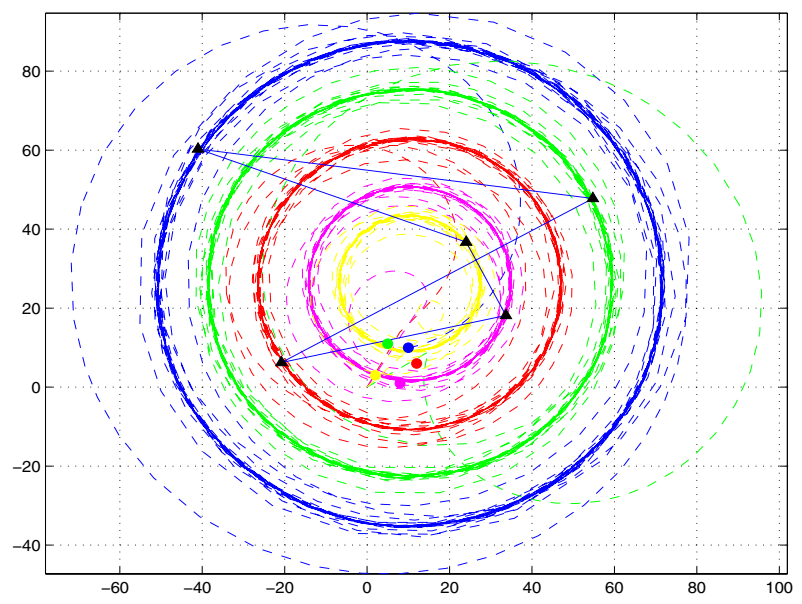

Fig. 13. Trajectories of $n=5$ agents for Case 3 (circle: initial position, triangle: final position of the UAVs)

$\rho_{2}, \rho_{3}$ and $\rho_{4}$ have non-empty intersection. The ranges of $\rho$ for which $f(\rho)$ exist is $50 \leq \rho \leq 54$ as shown in Fig. 9 . We plot $f(\rho)$ for this range of $\rho$ in Fig. 11. There is no value of $\rho$ or $q$ that satisfies (8). The simulation result is shown in Fig. 10 and reveals that this case indeed does not have an equilibrium. This shows that the existence of $\rho$ that satisfies (12) is not sufficient for (8) to have a solution.

Case 3: Again, consider the previous example, but now use $V_{5}=7$. The overlapping region of $\rho$ for $m=0$ is $50 \leq \rho \leq 63$. The range of $\rho$ and $f(\rho)$ are shown in Fig. 12 and Fig. 11, respectively. At $\rho=61.7$, (8) is satisfied. This gives the radius of other agents (from (5)) as $\rho=\left[\begin{array}{lllll}61.7 & 49.3 & 37.0 & 27.7 & 17.3\end{array}\right]$. The simulation, shown in Fig. 14, confirms the radius of the circles evaluated analytically.

Case 4. When the necessary condition (9) is satisfied, (8)

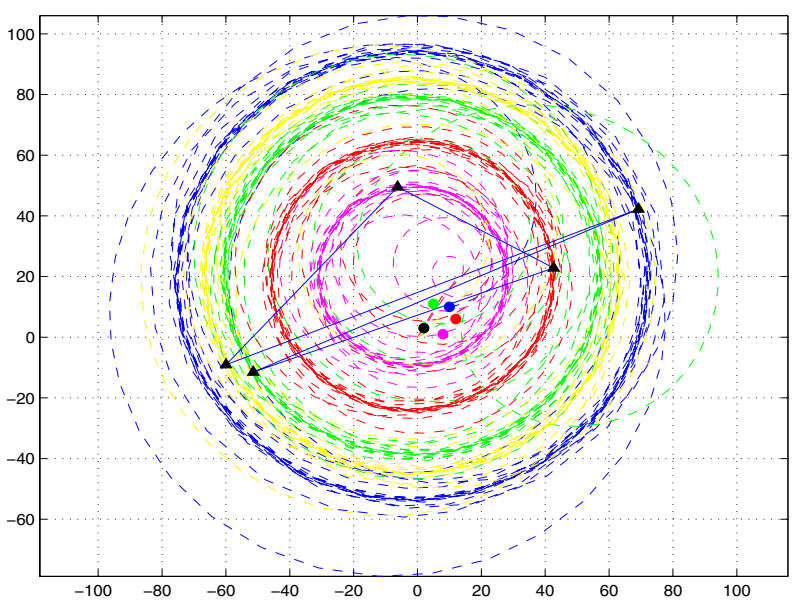

Fig. 14. Trajectories of $n=5$ agents for case 4 (stable equilibrium) (circle: initial position, triangle: final position of the UAVs)
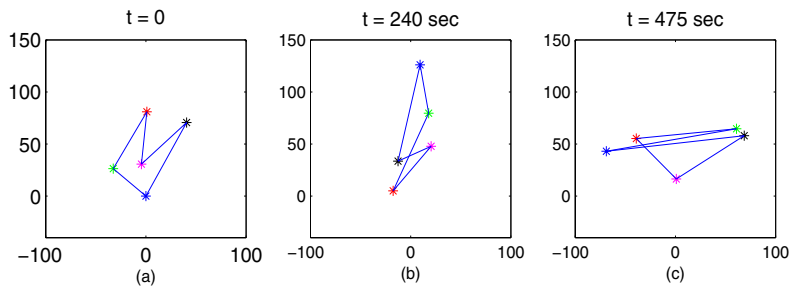

Fig. 15. Trajectories of $n=5$ agents showing unstable equilibrium (a) initial equilibrium configuration, (b) intermediate configuration, (c) final stable configuration corresponding to $q=2$

can have more than one solution depending on the value of $q$. For example, again consider the previous case (Case 1) but now $V_{5}=22, X_{1}=\left[\begin{array}{lll}1 & 2 & 3\end{array}\right]$. The range of values of $\rho$ is shown in Fig. 16 and $f(\rho)$ is plotted in Fig. 11. From the figure it can be seen that more than one solution $(\rho=50.9$ and 74.0) exists for this particular problem.

However, $\rho=50.9$ is unstable. Taking $R_{1}=\rho=50.9$, and assuming equilibrium values $\alpha_{1}=0$, we get $\alpha=\left[\begin{array}{lllll}0 & -0.94 & 3.13 & -0.25 & 2.04\end{array}\right]$ and $\theta=\left[\begin{array}{lllll}2.46 & 1.02 & 4.60 & 0.73 & 4.20\end{array}\right]$ at equilibrium. These values satisfies $\dot{r}_{i}=0, \ddot{\alpha}_{i}=0$ (implying $\dot{\alpha}_{i}=$ constant), and $\ddot{\theta}_{i}=0$ (implying $\dot{\theta}_{i}=$ constant), for all $i$. Therefore, this corresponds to a equilibrium point. However, simulation shows that even if we start from this equilibrium point, due to numerical errors, it slowly migrates to the other equilibrium point at $\rho=74$. This is shown in Fig. 15.

Case 5: However, if more than one equilibrium point is stable, the system converges to one of the equilibrium formation depending on the initial configuration. For example, consider $V=\left[\begin{array}{llllll}20 & 18 & 16 & 14 & 12\end{array}\right]$ and $k=\left[\begin{array}{lllll}5 & 5 & 5 & 5 & 5\end{array}\right] . f(\rho)$ for this case is shown in Fig. 17. The final formation for two different initial conditions is shown in Fig. 18. The dependence of the final configuration on initial configuration has also been observed in [5].

We further conjecture that at equilibrium, all agents 


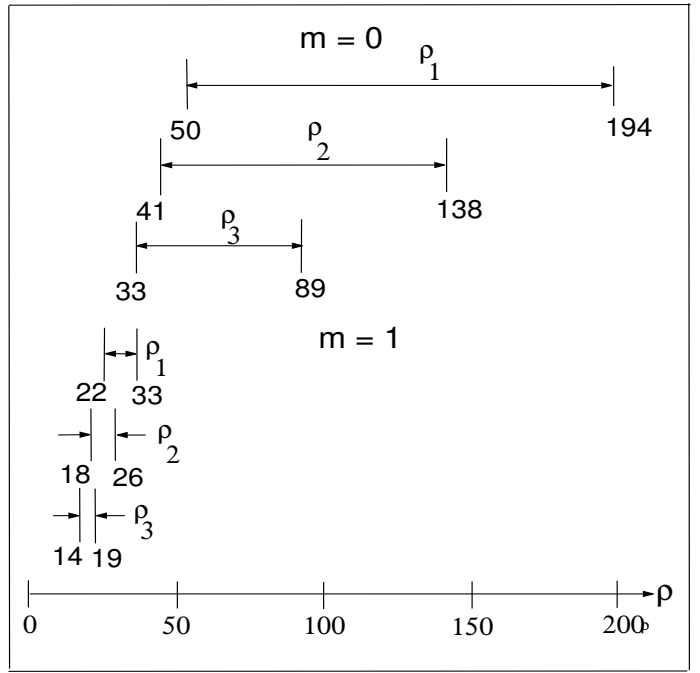

Fig. 16. Range of $\rho_{i}$ for Case 4

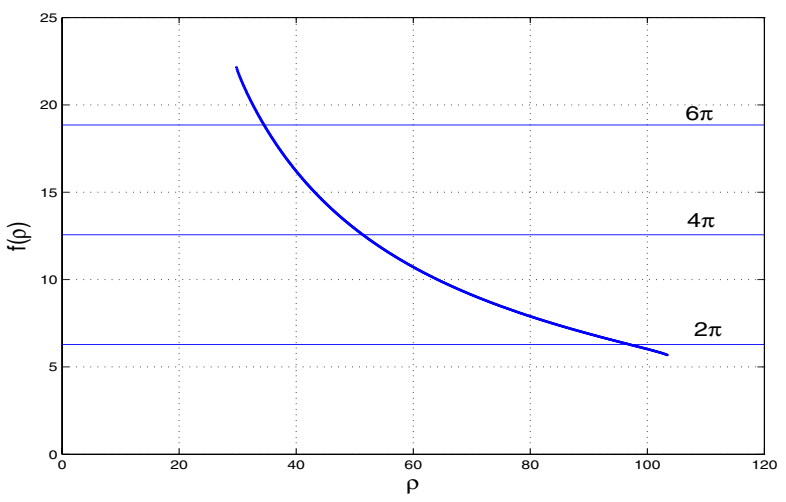

Fig. 17. Roots of Eqn (8) for Case 5

move in counter-clockwise direction, therefore, the condition $\bigcap_{i \in X_{1}} \mathcal{R}_{i} \neq \emptyset$, from Eqn. (12), occurs only at $m=0$. This simplifies the effort required to find the overlapping region. We hope to prove this conjecture along with the stability results.

\section{CONCLUSION}

Cyclic pursuit strategies has recently been of much interest among researchers. In this paper, the formation for a group of heterogenous mobile agents (with different speeds and controller gains) is studied. A necessary condition and its refinements are presented that generalizes several of the results given in [5] and gives analytical expressions for computing the radius of the circles and relative angles between agents at equilibrium.

\section{REFERENCES}

[1] A.M. Bruckstein, N. Cohen and A. Efrat, Ants, crickets and frogs in cyclic pursuit, Technical Report 9105, Center for Intelligence Systems, Technion-Israel Institute of Technology, Haifa, Israel July1991.

[2] Z. Lin, M. Broucke and B. Francis, Local control strategies for groups of mobile autonomous agents, Proceedings of the IEEE Conference on Decision and Control, Maui, Hawaii, December 2003, pp. 10061011
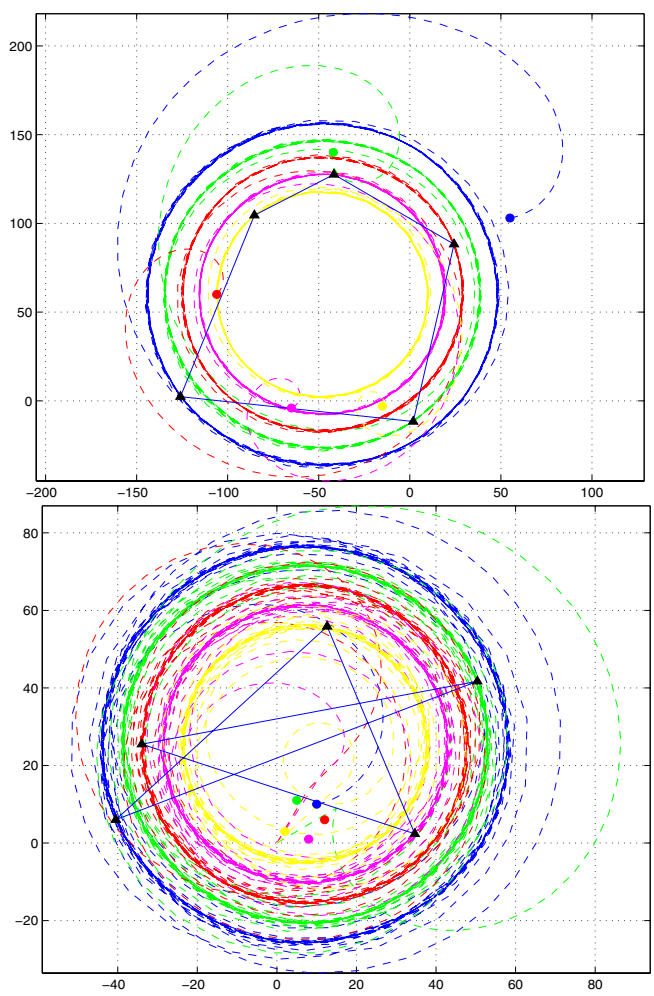

Fig. 18. Trajectories of $n=5$ agents for case 5 for different initial conditions (circle: initial position, triangle: final position of the UAVs)

[3] J.A. Marshall, M.E. Broucke, B.A. Francis, Pursuit strategies for wheeled-vehicle formations, Proceedings of the IEEE Conference on Decision and Control, Maui, Hawaii, December 2003, pp. 2555-2560

[4] J.A. Marshall, M.E. Broucke, B.A. Francis, Unicycles in cyclic pursuit, Proceedings of the 2004 American Control Conference, Boston, Massachusetts, June 2004, pp. 5344-5349

[5] J.A. Marshall, M.E. Broucke, B.A. Francis, Formation of vehicles in cyclic pursuit, IEEE Transactions on Automatic Control, Nov 2004 (to appear)

[6] T. Richardson, Stable polygons of cyclic pursuit, Annals of Mathematical and Artificial Intelligence, vol. 31, pp. 147-172, Issue 1-4 2001.

[7] T.J. Richardson, Non-mutual captures in cyclic pursuits, Annals of Mathematical and Artificial Intelligence, vol. 31, pp. 127-146, Issue 1-4 2001.

[8] F. Behroozi, R. Gagnon, Cyclic pursuit in a plane, Journal of Mathematical Physics, vol. 20, pp. 2212-2216, Nov 1979.

[9] M.S. Klamkin and D.J. Newman, Cyclic pursuit or "the three bugs problem", The American Mathematical Monthly, Vol. 78, No. 6, pp. 631-639, June-July 1971.

[10] A. Bernhard, Polygons of pursuit, Scripta Mathematica, Vol. 24, pp. 23-50, 1959.

[11] A. Sinha and D. Ghose, Some generalization of linear cyclic pursuit, Proceedings of IEEE India Annual Conference 2004, INDICON 2004, Kharagpur, India, December 2004, pp. 210-213. 